\title{
Delirio postoperatorio en pacientes neuroquirúrgicos: evaluación mediante el Test
}

\section{Mental Abreviado}

\author{
J. Hernández-Palazón; P. Doménech-Asensi; M.A. Pérez-Espejo*; F. López-Hernández*; S. Burguillos-López y
} J.F. Martínez-Lage*

Servicio de Anestesiología y Reanimación.*Servicio Regional de Neurocirugía. Hospital Universitario Virgen de la Arrixaca. Murcia.

Resumen

Objetivo. Evaluar la incidencia y características de la alteración postoperatoria de las funciones cerebrales superiores en los pacientes sometidos a cirugía intracraneal electiva bajo anestesia general.

Pacientes y método. Estudio prospectivo de 60 pacientes ASA I-III, de edades comprendidas entre los 18 y 81 años, intervenidos quirúrgicamente por el Servicio de Neurocirugía de nuestro Hospital, distribuidos en dos grupos de 30 pacientes cada uno: grupo cirugía intracraneal, pacientes sometidos a cirugía por procesos intracraneales, y grupo cirugía extracraneal o grupo control, pacientes intervenidos de columna cervical o lumbar. Se registraron los datos demográficos de edad, sexo, peso, talla, así como hábitos, enfermedades coexistentes y medicación habitual. A todos los pacientes se les realizó el test mental abreviado (AMT) el día previo a la intervención quirúrgica y a las 2 y 24 horas del periodo postoperatorio. Todos los pacientes fueron sometidos a la misma técnica anestésica. Fueron excluidos los pacientes que presentaron un AMT preoperatorio $\leq 8$.

Resultados. No hubo diferencias en los datos demográficos, duración de la cirugía, estado físico ASA y consumo de alcohol y tabaco. No se hallaron diferencias significativas entre grupos en la puntuación media obtenida en el AMT realizado el día antes de la cirugía, que fue de 9,87 $\pm 0,35$ para los pacientes sometidos a cirugía intracraneal y de $\mathbf{9 , 8 0} \pm \mathbf{0 . 4 1}$ para los pacientes intervenidos de columna. Así mismo, no se observaron diferencias significativas entre los dos grupos en el AMT realizado a la 2 y 24 horas del postoperatorio: 4 de los pacientes sometidos a cirugía intracraneal presentaron una puntuación en el AMT $\leq 8$ a las 2 horas de finalizada la cirugía, y 3 pacientes del mismo grupo, a las 24 horas del postoperatorio; tan solo 2 pacientes intervenidos de columna presentaron una puntuación en el AMT $\leq \mathbf{8}$ a las $\mathbf{2}$ horas de finalizada la cirugía y ninguno a las

Recibido: 28-02-05. Aceptado: 17-05-05
24 horas del postoperatorio.

Conclusiones. Según los resultados obtenidos en este estudio podemos concluir que los pacientes sometidos a cirugía intracraneal no sufren alteraciones de la función cognitiva ni de la atención durante las primeras 24 horas del postoperatorio, evaluados mediante el AMT.

PALABRAS CLAVE. Delirium postoperatorio. Test Mental Abreviado. Anestesia general. Neurocirugía. Craneotomía.

Postoperative delirium in patient neurosurgical: evaluation by means of the Abbreviated Mental Test

\section{Summary}

Objectives. To assess the incidence and characteristics of postoperative changes in the higher cerebral functions after elective intracranial surgery under general anesthesia.

Patients and methods. This is a prospective study of 60 patients, aged 18-81 years, submitted to neurosurgical operations, allocated into two groups of 30 patients each: intracranial surgery group, patients submitted to craniotomy, and extracranial surgery group or control group, patients submitted to spinal procedures. All patients were given the Abbreviated Mental Test (AMT) on the day before to the operation, and then 2 and 24 hours after the end of the procedure. All individuals were managed with the same anesthetic technique.

Results. No differences in regard to demographics, duration of the operation, ASA physical state, and habits were found between the two groups. No differences in the pre-surgical and post-surgical AMT mean score were encountered between patients submitted to intracranial $9.87 \pm 0.35$ or to spinal surgeries $9.80 \pm$ 0.41. Similarly, there were no significant differences between the two groups in the results of the AMT performed at 2 and 24 hours after the end of the surgeries.

Abreviaturas. ASA: American Society of Anesthesiologist. AMT: Abbreviated Mental Test. 
Only two subjects undergoing spinal procedures had a score of $\leq 8$ in the AMT performed 2-hours after the operation, while none showed a decrease in the 24-hour test score.

Conclusions. Patients submitted to intracranial surgery did not show any changes in cognitive or attention functions during the first postoperative 24 hours as assessed by the AMT.

KEY WORDS. Postoperative delirium. Abbreviated mental test. General anesthesia. Neurosurgery. Craniotomy.

\section{Introducción}

El delirium postoperatorio es un síndrome ampliamente descrito a lo largo de los años, a pesar de lo cual persiste el problema de la terminología a la hora de definirlo: estado confusional agudo, síndrome orgánico cerebral, psicosis tóxica ${ }^{15}$, son algunas de las formas que siguen empleándose para su denominación. Este trastorno mental transitorio consiste en la alteración de las funciones cerebrales superiores, que se caracteriza por disminución de la capacidad de atención y alteración de las funciones cognitivas tales como lenguaje y orientación temporoespacial. Se ha distinguido entre delirium "de emergencia", refiriéndose al que se desarrolla en las primeras 24 horas del postoperatorio y el delirium "de intervalo", que se manifiesta tras uno o más días de intervalo lúcido tras la cirugía ${ }^{15}$. El delirium es fácilmente reconocible en pacientes con estado agudo de agitación; sin embargo, se requiere un elevado grado de sospecha en la mayoría de los pacientes sometidos a cirugía, para que este cuadro no pase desapercibido. Los tests mentales a pie de cama son útiles para detectar los cambios en la función mental y, por tanto, permiten diagnosticar precozmente el delirium postoperatorio. El Test Mental Abreviado (AMT) o test de Hodkinson's es un test ampliamente utilizado para valorar el estado mental del paciente quirúrgico. Originariamente, se diseñó para la valoración del estado mental de pacientes hospitalizados $y$, en la actualidad, se utiliza también para cuantificar las alteraciones de la función cognitiva y de la atención en pacientes quirúrgicos, debido a su fácil realización incluso en personas enfermas ${ }^{8}$. Consiste en un cuestionario de 10 preguntas que exploran la orientación temporoespacial, la atención y la memoria, en el que cada respuesta correcta es un punto ${ }^{4}$. Chonchubhair et $\mathrm{al}^{1}$ demostraron que un descenso de dos puntos o más en el resultado postquirúrgico, con relación al prequirúrgico, es un indicador sensible y específico de delirium en pacientes intervenidos.

Se han realizado múltiples estudios prospectivos para determinar la incidencia de delirium en la población quirúrgica en general. Sin embargo, no existen estudios que evaluen la magnitud o patrón de disfunción cognitiva postoperatoria en pacientes sometidos a craneotomía por patología intracraneal. Por este motivo, diseñamos este estudio para evaluar la incidencia y características de la alteración postoperatoria del estado mental en pacientes sometidos a cirugía intracraneal electiva bajo anestesia general.

\section{Pacientes y métodos}

Con la aprobación del Comité de Ética del Hospital realizamos un estudio clínico controlado en 60 pacientes de ambos sexos, estado físico ASA I-III y edades comprendidas entre los 18 y 81 años, sometidos a procedimientos neuroquirúrgicos bajo anestesia general. Los pacientes fueron distribuidos en dos grupos de 30 pacientes cada uno: a) pacientes objeto del estudio sometidos a craneotomía por procesos intracraneales (grupo cirugía intracraneal), y b) pacientes neuroquirúrgicos programados para cirugía de columna cervical o lumbar (grupo cirugía extracraneal o grupo control). Se realizaron 18 craneotomías para exéresis de tumor supratentorial, 7 para clipaje de aneurisma cerebral y 5 craniectomías de fosa posterior. La cirugía extracraneal incluyó 17 intervenciones de exéresis de disco y fusión vertebral cervical y 13 de fusión lumbar.

Se registraron variables de carácter sociodemográficas como edad, sexo, consumo de tabaco y alcohol. Así mismo, se obtuvieron datos relacionados con enfermedades asociadas, medicación habitual, estado físico ASA, tipo de intervención quirúrgica y estancia en reanimación. Todos los pacientes incluidos en el estudio fueron sometidos a la misma técnica anestésica, presentando antes de la intervención quirúrgica una puntuación de la escala de coma de Glasgow de 15. La inducción anestésica consistió en la administración de $0,02 \mathrm{mg} / \mathrm{kg}$ de midazolam, perfusión de $0,5 \mu \mathrm{g} / \mathrm{kg} / \mathrm{min}$ de remifentanilo durante el tiempo de inducción, $3-5 \mathrm{mg} / \mathrm{kg}$ de tiopental y $0,2 \mathrm{mg} / \mathrm{kg}$ cisatracurio. El mantenimiento anestésico se consiguió con perfusión de remifentanilo a $0.25-0.4 \mu \mathrm{g} / \mathrm{k} / \mathrm{g} / \mathrm{min}$, sevofluorano telespiratorio entre $0.8 \%-1 \%$ y perfusión de cisatracurio a $0,08 \mathrm{mg} / \mathrm{kg} / \mathrm{h}$. Al finalizar la intervención se revirtió el bloqueo neuromuscular con $2 \mathrm{mg}$ de neostigmina y $1 \mathrm{mg}$ de atropina.

Para analgesia postoperatoria se administró $1 \mathrm{~g}$ de paracetamol intravenoso y $0.1 \mathrm{mg} / \mathrm{kg}$ de cloruro mórfico $30 \mathrm{~min}$ antes del final de la cirugía. La profilaxis gástrica y antiemética se llevó a cabo con ondansetrón y ranitidina.

El estado mental de los pacientes incluidos en el estudio fue evaluado por medio de la aplicación del AMT (Tabla 1) el día previo a la cirugía y a las 2 y 24 horas de finalizada la intervención. El AMT consiste en un cuestionario de 10 preguntas que exploran la orientación temporoespacial, la atención y la memoria. Cada respuesta correcta en el test equivale a un punto. Dos de las preguntas del AMT 
Tabla 1

Test Mental Abreviado

1. Edad

2. Hora aproximada

3. Dirección para recordar al final del test: Plaza de España 42

4. Año

5. Nombre del Hospital

6. Reconocer dos personas (médico y enfermera)

7. Fecha de nacimiento

8. Año de comienzo de la Guerra Civil Española

9. Nombre del Rey

10. Contar hacia atrás de 20 a 1

exploran la habilidad individual para dar información sobre sí mismo ("¿Qué edad tiene?" y "¿Cuál es su fecha de nacimiento?"). Otras dos preguntas exploran la percepción temporal ("¿En qué año estamos?" y "¿Qué hora es?"). La orientación espacial se evalúa por otras dos cuestiones ("¿Cómo se llama este lugar?" y ¿¿Reconoce a estas personas?"). Tres preguntas valoran la memoria individual ("¿Puede repetir la dirección que le doy?", „¿En qué año comenzó la Guerra Civil Española?" y "¿Cómo se llama el Rey?"). Por último, una pregunta valora la capacidad de concentración y la habilidad de cálculo ("Cuente hacia atrás del 20 al 1"). En nuestro estudio consideramos que los pacientes con una puntuación $\leq 8$ puntos, presentaban un deterioro de la función mental ${ }^{1}$. Fueron excluidos del estudio los pacientes que presentaron una puntuación en el AMT preoperatorio $\leq 8$ puntos, enfermedad psiquiátrica, imposibilidad de realizar el test por afasia o sordera, incidentes anestésicos y/o quirúrgicos y negativa a la realización del test.

Los datos se analizaron mediante el programa estadístico SPSS para Windows. Los resultados se presentan como media \pm desviación estándar. Como tratamiento estadístico se utilizó el análisis de la varianza y la prueba de la t de Student para el análisis de variables cuantitativas y la prueba de la $\chi^{2}$ para las cualitativas. Se consideraron significativos los valores de $\mathrm{p}<0,05$.

\section{Resultados}

Los parámetros demográficos de edad y sexo, estado físico ASA, hábitos tóxicos, duración de la cirugía y estancia en reanimación se muestran en la Tabla 1. No se encontraron diferencias estadísticamente significativas entre los grupos estudiados en cuanto a edad, sexo, duración de la cirugía, consumo de tabaco y de alcohol. El porcentaje de pacientes con estado físico ASA II y III fue significativamente mayor $(p<0,001)$ en el grupo cirugía intracraneal que en el grupo de pacientes sometidos a cirugía de columna (Tabla 2). Así mismo, la estancia en reanimación fue significativamente mayor $(p<0,001)$ en los pacientes sometidos a cirugía intracraneal que en los pacientes del grupo cirugía extracraneal (Tabla 2).

No hubo diferencias significativas en el AMT realizado entre los dos grupos antes de la cirugía ni a las 2 y 24 horas del postoperatorio (Tabla 3). Los pacientes intervenidos para cirugía intracraneal presentaron una puntuación tras

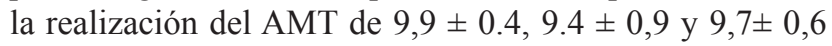
previo a la cirugía y a las 2 y 24 horas, respectivamente, frente a los pacientes intervenidos de columna, que registraron una puntuación de $9,8 \pm 0,4,9.6 \pm 0,5$ y $9,8 \pm 0,4$ previo a la cirugía y a las 2 y 24 horas del postoperatorio, respectivamente. Cuatro (13\%) pacientes del grupo cirugía intracraneal presentaron una puntuación en el AMT $\leq 8$ a las 2 horas de finalizada la cirugía, y $3(10 \%)$ pacientes a las 24 horas del postoperatorio, y tan solo $2(7 \%)$ pacientes intervenidos de columna mostraron una puntuación en el $\mathrm{AMT} \leq 8$ a las 2 horas de finalizada la cirugía y ninguno

\section{Tabla 2}

Características demográficas, hábitos tóxicos, ASA, duración de la cirugía y estancia en reanimación

\begin{tabular}{lcc}
\hline & Cirugía intracraneal & Cirugía extracraneal \\
\hline Edad (años) & $42 \pm 18$ & $45 \pm 15$ \\
Sexo (H/M) & $14 / 16$ & $7 / 23$ \\
ASA (\%) & I (17) / II (76) / III (7) & I (53) / II (23) / III (23)* \\
Fumador / No fumador (\%) & $50 / 50$ & $37 / 63$ \\
Bebedor / No bebedor (\%) & $53 / 47$ & $46 / 54$ \\
Duración cirugía (min) & $298 \pm 48$ & $272 \pm 72$ \\
Estancia en Reanimación (horas) & $17,5 \pm 6,6$ & $6,2 \pm 1,6 \dagger$ \\
\hline
\end{tabular}

$*=$ Prueba de la $\chi^{2} p<0,001 ; \dagger=$ Prueba de la $\mathbf{t}$ de Student $p<0,001$ 
Tabla 3

Puntuación media del AMT aplicado el día antes de la cirugía y a las 2 y 24 horas del postoperatorio

\begin{tabular}{lcc}
\hline & Cirugía intracraneal & Cirugía extracraneal \\
\hline Preoperatorio & $9,9 \pm 0,4$ & $9,8 \pm 0,4$ \\
Postoperatorio $(2 \mathrm{~h})$ & $9,4 \pm 0,9$ & $9,6 \pm 0,5$ \\
Postoperatorio $(24 \mathrm{~h})$ & $9,7 \pm 0,6$ & $9,8 \pm 0,4$
\end{tabular}

Valores expresados como media \pm desviación estándar.

Tabla 4

Respuestas favorables al Test Mental Abreviado

\begin{tabular}{lcccccc}
\hline & \multicolumn{2}{c}{ Preoperatorio } & \multicolumn{2}{c}{ Postoperatorio $(2 \mathrm{~h})$} & \multicolumn{2}{c}{ Postoperatorio $(24 \mathrm{~h})$} \\
\hline & Intracraneal Extracraneal & Intracraneal Extracraneal & Intracraneal Extracraneal \\
\hline Edad & 30 & 30 & 30 & 30 & 30 & 30 \\
Hora aproximada & 28 & 30 & 29 & 19 & 28 & 24 \\
Dirección para recordar al final: Plaza de España 42 & 30 & 24 & 25 & 30 & 27 & 30 \\
Año & 30 & 30 & 29 & 30 & 28 & 30 \\
Nombre del Hospital & 30 & 30 & 30 & 30 & 30 & 30 \\
Reconocer dos personas (médico y enfermera) & 30 & 30 & 30 & 30 & 29 & 30 \\
Fecha de nacimiento & 30 & 30 & 30 & 30 & 30 & 30 \\
Año de comienzo de la Guerra Civil Española & 28 & 30 & 30 & 30 & 30 & 30 \\
Nombre del Rey & 30 & 30 & 30 & 30 & 30 & 30 \\
Contar hacia atrás de 20 a 1 & 30 & 30 & 28 & 30 & 30 & 30 \\
\hline
\end{tabular}

a las 24 horas del postoperatorio. El número de respuestas acertadas por los pacientes de cada grupo a cada una de las 10 preguntas tras la aplicación del AMT se muestran en la Tabla 4.

\section{Discusión}

A pesar de los avances en las técnicas quirúrgicas y anestésicas, el estado confusional agudo reversible o delirium sigue siendo en la actualidad una de las complicaciones postquirúrgicas más frecuentes, sobre todo en la población adulta de mayor edad ${ }^{14}$. En nuestro estudio, evaluamos la incidencia de delirium de emergencia, es decir, el que se manifiesta en las primeras 24 horas del postoperatorio, en pacientes neuroquirúrgicos. Coincidiendo con estudios prospectivos de población quirúrgica general, sin límite de edad, la tasa de incidencia global de delirium postoperatorio en nuestro estudio fue del $10 \%{ }^{15}$. Además, en nuestro estudio diferenciamos los pacientes que fueron operados de craneotomía por procesos intracraneales de los pacientes que fueron intervenidos de fusión vertebral. A diferencia de otros estudios donde observaron que el riesgo de delirium postoperatorio aumentaba con la complejidad del proceso quirúrgico ${ }^{10,15,18}$, en nuestro estudio realizado en pacientes neuroquirúrgicos, el tipo de cirugía no constituyó un factor precipitante en la aparición de disfunción cognitiva, ya que no se encontraron diferencias significativas en la puntuación media del AMT a las 2 y 24 horas del postoperatorio en los pacientes estudiados. Sin embargo, existieron diferencias cualitativas en la realización del AMT en relación con el tipo de cirugía, ya que los pacientes sometidos a craneotomía presentaron alteraciones de la memoria individual, capacidad de concentración y de la percepción temporal a diferencia de los pacientes intervenidos de columna que sólo presentaron alteraciones de la percepción temporal, posiblemente debido a una mayor estancia en la unidad de reanimación de los pacientes del grupo de cirugía intracraneal.

Según algunos autores ${ }^{2,9-11,15,19}$, la incidencia de delirium tras la cirugía va a depender de la edad del paciente, enfermedades médicas asociadas, función cognitiva preoperatoria, fármacos utilizados para la anestesia, tipo de 
cirugía así como del método diagnóstico utilizado para el diagnóstico del delirium ${ }^{2}$. La edad avanzada se asocia con el riesgo más alto de delirium en el postoperatorio ${ }^{9}$, ya que los pacientes ancianos son susceptibles de alteraciones del estado mental tras la cirugía como resultado de los cambios físicos asociados con el proceso de envejecimiento normal, sobre todo a nivel cerebral ${ }^{15}$. Además, la capacidad de regulación de la homeostasia en los ancianos frente a la agresión quirúrgica y/o anestésica está disminuida, aumentando la probabilidad de la aparición de efectos secundarios tras la administración de dosis terapéuticas de determinados fármacos, que podrían precipitar la aparición de delirium en estos pacientes ${ }^{19}$. La existencia de un mal estado funcional preoperatorio asociado a la existencia de enfermedades médicas, se ha relacionado con una mayor incidencia de delirium o alteraciones de la función cognitiva en el postoperatorio ${ }^{11}$. Una mención especial merecen los pacientes con cáncer o aquéllos que presentan enfermedades estructurales cerebrales como los pacientes con enfermedad de Parkinson, enfermedad cerebrovascular o síndrome depresivo que presentan una incidencia mayor de delirium en el postoperatorio que el observado en la población no afecta ${ }^{5,11,13,17}$. Respecto a la técnica anestésica, no se ha puesto de manifiesto en estudios recientes que el tipo de anestesia afecte la función cognitiva postoperatoria, no encontrándose diferencias significativas en la incidencia de delirium postoperatorio en la anestesia regional en comparación con la general ${ }^{12,19}$. No obstante, el riesgo de delirium aumenta con la complejidad del procedimiento quirúrgico, si bien no se puede asumir que se deba a los efectos de la cirugía o de la anestesia, existiendo publicaciones recientes que demuestran la relación causal entre las complicaciones médicas o quirúrgicas postoperatorias y la aparición de delirium ${ }^{10,16}$.

El delirium es fácilmente reconocible en pacientes con estado agudo de agitación, pero a menudo es mal interpretado o mal diagnosticado debido a la variabilidad de sus síntomas. Por este motivo es fundamental en el diagnóstico cuantificar las alteraciones de la función cognitiva y de la atención por medio de la realización de un test de evaluación de la función mental en los pacientes quirúrgicos. El AMT es el test para la estimación del estado mental más frecuentemente utilizado en los pacientes quirúrgicos debido a su simplicidad y brevedad ${ }^{1}$. Está especialmente indicado en ancianos y en personas enfermas porque las preguntas que incluye pueden contestarse sin escribir, leer o dibujar; de este modo, las dificultades de visión o escritura que presentan muchos pacientes no alteran la realización del test. Un descenso de dos puntos o más en el resultado postquirúrgico con relación al prequirúrgico es un indicador sensible y específico de delirium en pacientes intervenidos ${ }^{1}$.

Se han descrito como posibles factores de riesgo para el desarrollo de disfunción cognitiva postoperatoria las alteraciones estructurales cerebrales presentes en pacientes ancianos $^{12}$, la hipotensión intraoperatoria ${ }^{3}$, la hipoxemia perioperatoria ${ }^{7}$ y las complicaciones postoperatorias ${ }^{6}$. Los pacientes sometidos a cirugía intracraneal por enfermedad cerebral deberían ser más vulnerables a los efectos perioperatorios de la hipoxemia e hipotensión arterial, ya que estos pacientes pueden presentar una alteración de la autorregulación cerebral y la aparición de delirium de emergencia podría estar provocada por la disminución de la presión de perfusión cerebral y de la oxigenación cerebral. Sin embargo, en nuestro estudio no hubo complicaciones quirúrgicas ni anestésicas significativas, así como no se observaron episodios de hipoxemia grave o de hipotensión arterial durante la intervención quirúrgica ni en el postoperatorio inmediato; esto justificaría la escasa incidencia de disfunción cognitiva postoperatoria tanto en los pacientes intervenidos de craneotomía/craniectomía como en los de fusión vertebral. Desde el punto de vista metodológico, el pequeño tamaño de la muestra supone una limitación a este estudio clínico, ya que no permite conocer la influencia que podrían tener en los resultados diferentes variables, tales como la edad, tipo de lesión intracraneal, enfermedades asociadas, medicación habitual, y estado físico ASA.

De los resultados obtenidos en este estudio, podemos concluir que la cirugía intracraneal no se asocia con un elevado riesgo de delirium postoperatorio, ya que no se afectaron significativamente la función mental ni la atención en los pacientes estudiados tras la aplicación del AMT. Sin embargo, hay que tener en cuenta que el delirium sigue siendo una complicación frecuente en el postoperatorio, sobre todo en el paciente anciano, siendo responsable de una estancia hospitalaria prolongada, disminución de la capacidad funcional y mayor frecuencia de alta hospitalaria a otros centros de larga estancia, por lo que se recomienda la utilización de un test de evaluación de la función mental de fácil realización como el AMT para el diagnóstico de las alteraciones de la función mental, además de una estrecha colaboración entre el equipo médico y de enfermería para mantener la orientación del paciente y disminuir la incidencia de delirium postoperatorio en el paciente neuroquirúrgico.

\section{Bibliografía}

1. Chonchubhair, A.Ní., Valacio, R., Kelly, J., O'Keeffe, S.: Use of the abbreviated mental test to detect postoperative delirium in elderly people. Br J Anaesth 1995; 75: 481-482.

2. Dyer, C.B., Ashton, C.M., Teasdale, T.A.: Postoperative delirium. A review of 80 primary data-collection studies. Arch Intern Med 1995; 155: 461-465.

3. Edlund, A., Lundstrom, M., Brannstrom, B., et al.: Delirium 
before and after operation for femoral neck fracture. J Am Geriatr Soc 2001; 49: 1335-1340.

4. Flicker, I., Logiudice, D., Carlin, J.B., Ames, D.: The predictive value of dementia screening instruments in clinical populations. Int J Geriatr Psychiatry 1997; 12: 203-209.

5. Golden, W.E., Lavender, R.C., Metzer, W.S.: Acute postoperative confusion and hallucinations in Parkinson disease. Ann Intern Med 1989; 111: 218-222.

6. Gustafson, Y., Berggren, D., Brannstrom, B., et al.: Acute confusional state in elderly patients treated for femoral neck fracture. J Am Geriatr Soc 1988; 36: 525-530.

7. Gustafson, Y., Brannstrom, B., Berggren, D., et al.: A geriatric -anesthesiologic program to reduce acute confusional states in elderly patients treated for femoral neck fractures. J Am Geriatr Soc 1991a; 39: 655-662.

8. Hodkinson, H.M.: Evaluation of a mental test score for assessment of mental impairment in the elderly. Age Ageing 1971; 1: 233-238.

9. Lipowsky, Z.J.: Delirium in the elderly patient. N Engl J Med 1989; 320: 578-582.

10. Lynch, N.M., Trousdale, R.T., Ilstrup, D.M.: Complications after concomitant bilateral total knee artroplasty in elderly patients. Mayo Clin Proc 1997; 72: 799-805.

11. Marcantonia, E.R., Goldman, L., Mangione, C.M., et al.: A clinical prediction rule for delirium after elective noncardiac surgery. JAMA 1994; 271: 134-139.

12. Moller, J.T., Cluitmans, P., Rasmussen, L.S., et al.: Long-term postoperative cognitive dysfunction in the elderly. Lancet 1998; 351: 857-861.
13. Morse, R.M., Litin, E.M.: Postoperative delirium: a study of etiologic factors. Am J Psychiatry 1969; 126: 388395.

14. Newman, M.F., Kirchner, J.L., Phillips-Bute, B., et al.: Longitudinal assessment of neurocognitive function after coronary artery bypass surgery. N Engl J Med 2001; 344: 395402 .

15. O'Keeffe, S.T., Chonchubhair, A.Ní.: Postoperative delirium in the elderly. Br J Anaesth 1994; 73: 673-687.

16 . O'Keeffe, D., Lavan, J.: The prognostic significance of delirium in older hospital patients. J Am Geriatr Soc 1997; 45: 174-178.

17. Silverfarb, P.M.: Chemotherapy of cognitive deficits in cancer patients. Annu Rev Med 1983; 34: 35-45.

18. Schor, J.D., Levkoff, S.E., Lipsitz, L.A.: Risk factors for delirium in hospitalised elderly. JAMA 1992; 267: 827831 .

19. Williams-Russo, P., Sharrock, Ne., Mattis, S.A., et al.: Cognitive effects after epidural vs general anesthesia in older adults. A randomized trial. JAMA 1995; 274: 44-50.

Hernández-Palazón, J.; Doménech-Asensi, P.; PérezEspejo, M.A.; López-Hernández, F.; Burguillos-López, S.; Martínez-Lage, J.F.: Delirio postoperatorio en pacientes neuroquirúrgicos: evaluación mediante el Test Mental Abreviado. Neurocirugía 2006; 17: 119-124.

Correspondencia postal: Joaquín Hernández Palazón. San Ignacio de Loyola, 4 - $2^{\circ}$ B. 30001 Murcia. 